TAIWANESE JOURNAL OF MATHEMATICS

Vol. 5, No. 3, pp. 519-533, September 2001

This paper is available online at http://www.math.nthu.edu.tw/tjm/

\title{
A SPACE OF MEROMORPHIC MAPPINGS AND AN ELIMINATION OF DEFECTS
}

\author{
Seiki Mori*
}

\begin{abstract}
This is a summary report of my recent articles. Nevanlinna theory asserts that each meromorphic mapping $f$ of $\mathbb{C}^{m}$ into $\mathbb{P}^{n}(\mathbb{C})$ has few defects. However, it seems that meromorphic mappings with defects are very few. In this report, we shall show that for any given transcendental meromorphic mapping of $\mathbb{C}^{m}$ into $\mathbb{P}^{n}(\mathbb{C})$, there is a small deformation of $f$ which has no Nevanlinna deficient hyperplanes in $\mathbb{P}^{n}(\mathbb{C})$, and also in the case $m=1$, there is a small deformation of $f$ which has no Nevanlinna deficient hypersurfaces of degree $\leq d$ for each given positive integer $d$, or deficient rational moving targets. Furthermore, we shall show that mappings without Nevanlinna defects are dense in a space of transcendental meromorphic mappings.
\end{abstract}

\section{INTRODUCTION}

Nevanlinna defect relations were established for various cases, for example, holomorphic (or meromorphic) mappings of $\mathbb{C}^{m}$ into a complex projective space $\mathbb{P}^{n}(\mathbb{C})$ for constant targets of hyperplanes or moving targets of hyperplanes (arbitrary $m \geq 1$ and $n \geq 1$ ), or holomorphic mappings of an affine variety $A$ of dimension $m$ into a projective algebraic variety $V$ of dimension $n$ for divisors on $V(m \geq n \geq 1)$, and so on. On the other hand, the size of a set of Valiron deficient hyperplanes or deficient divisors are investigated (e.g., Sadullaev [8], Mori [4]). Nevanlinna theory asserts that for each holomorphic (or meromorphic) mapping, Nevanlinna defects or Valiron defects of the mapping are very few. Until now, there are few results on defects of a family of mappings. Recently, the author $[4,5,6]$ proved that for a transcendental meromorphic mapping $f$ of $\mathbb{C}^{m}$ into $\mathbb{P}^{n}(\mathbb{C})$, we can eliminate all

Received May 31, 2000; revised November 9, 2000.

Communicated by P. Y. Wu.

2000 Mathematics Subject Classification: 30D35, 32H30.

Key words and phrases: Nevanlinna theory, defect, meromorphic mapping.

* This research was partially supported by Grant-in-Aid for Scientific Research (No.10640149), Ministry of Education, Science and Culture of Japan. 
deficient hyperplanes $(m \geq 1)$, all deficient hypersurfaces of degree at most a given integer $d$ or rational moving targets $(m=1)$ in $\mathbb{P}^{n}(\mathbb{C})$ by a small deformation of the mapping. The class of meromorphic mappings which does not have a Valiron deficiency is important, because these functions have a counting function $N(r, D) \sim$ $T_{f}(r), r \rightarrow \infty$, for every target $D$.

We shall now discuss an elimination theorem of defects of a meromorphic mapping or a holomorphic curve by its small deformation, and also discuss a space of meromorphic mappings without defects. Here a small deformation $\tilde{f}$ of $f$ means that their order functions $T_{f}(r)$ and $T_{\tilde{f}}(r)$ satisfy $\left|T_{f}(r)-T_{\tilde{f}}(r)\right| \leq o\left(T_{f}(r)\right)$ as $r$ tends to infinity.

\section{Preliminaries}

\section{2-1. Notation and Terminology}

Let $z=\left(z_{1}, \ldots, z_{m}\right)$ be the natural coordinate system in $\mathbb{C}^{m}$. Set

$\langle z, \xi\rangle=\sum_{j=1}^{m} z_{j} \xi_{j}$ for $\xi=\left(\xi_{1}, \ldots, \xi_{m}\right),\|z\|^{2}=\langle z, \bar{z}\rangle, B(r)=\left\{z \in \mathbb{C}^{m} \mid\|z\|<r\right\}$,

$\partial B(r)=\left\{z \in \mathbb{C}^{m} \mid\|z\|=r\right\}, \quad \psi=d d^{c} \log \|z\|^{2}$ and $\sigma=d^{c} \log \|z\|^{2} \wedge \psi^{m-1}$, where $d^{c}=(\sqrt{-} 1 / 4 \pi)(\bar{\partial}-\partial)$, and $\psi^{k}=\psi \wedge \cdots \wedge \psi(k$-times).

Let $f$ be a meromorphic mapping of $\mathbb{C}^{m}$ into $\mathbb{P}^{n}(\mathbb{C})$. Then $f$ has a reduced representation $\left(f_{0}: \ldots: f_{n}\right)$, where $f_{0}, \ldots, f_{n}$ are holomorphic functions on $\mathbb{C}^{m}$ with $\operatorname{codim}\left\{z \in \mathbb{C}^{m} \mid f_{0}(z)=\cdots=f_{n}(z)=0\right\} \geq 2$. We write $f=\left(f_{0}, \ldots, f_{n}\right)$ as the same letter of the meromorphic mapping $f$. Denote $D^{\alpha} f=\left(D^{\alpha} f_{0}, \ldots, D^{\alpha} f_{n}\right)$ for a multi-index $\alpha$, where $D^{\alpha} \phi=\partial^{|\alpha|} \phi / \partial z_{1}^{\alpha_{1}} \cdots \partial z_{m}^{\alpha_{m}}, \alpha=\left(\alpha_{1}, \ldots, \alpha_{m}\right),|\alpha|=$ $\alpha_{1}+\cdots+\alpha_{m}$ and a function $\phi$.

Definition (see Fujimoto [2, §4]). We define the generalized Wronskian of $f$ by

$$
W_{\alpha^{0}, \ldots, \alpha^{n}}(f)=\operatorname{det}\left(D^{\alpha^{k}} f: 0 \leq k \leq n\right),
$$

for $n+1$ multi-indices $\alpha^{k}=\left(\alpha_{1}^{k}, \ldots, \alpha_{m}^{k}\right)(0 \leq k \leq n)$.

By Fujimoto [2, §4], for every linearly nondegenerate meromorphic mapping $f$ of $\mathbb{C}^{m}$ into $\mathbb{P}^{n}(\mathbb{C})$, there are $n+1$ multi-indices $\alpha^{0}, \ldots, \alpha^{n}$ such that $\left\{D^{\alpha^{0}} f, \ldots, D^{\alpha^{n}} f\right\}$ is an admissible basis with $\left|\alpha^{k}\right| \leq n+1$. Then $W_{\alpha^{0}, \ldots, \alpha^{n}}(\phi f)=\phi^{n+1} W_{\alpha^{0}, \ldots, \alpha^{n}}(f) \not \equiv$ 0 holds for any nonzero holomorphic function $\phi$ on $\mathbb{C}^{m}$, where $\phi f=\left(\phi f_{0}, \ldots, \phi f_{n}\right)$.

Let $f$ be a nonconstant meromorphic mapping $f$ of $\mathbb{C}^{m}$ into $\mathbb{P}^{n}(\mathbb{C})$, and let $\mathcal{L}=\left[H^{d}\right]$ be the line bundle over $\mathbb{P}^{n}(\mathbb{C})$ which is determined by the $d$ th tensor power of the hyperplane bundle $[H]$. A hypersurface $D$ of degree $d$ in $\mathbb{P}^{n}(\mathbb{C})$ is given by the divisor of a holomorphic section $\delta \in H^{0}\left(\mathbb{P}^{n}(\mathbb{C}), \mathcal{O}(\mathcal{L})\right)$ which is determined by a homogeneous polynomial $P(w)$ of degree $d$. A metric $a=\left\{a_{\alpha}\right\}$ 
on the line bundle $\mathcal{L}$ is given by $a_{\alpha}=\left(\sum_{j=0}^{n}\left|w_{j} / w_{\alpha}\right|^{2}\right)^{d}$ in a neighborhood $U_{\alpha}=\left\{w_{\alpha} \neq 0\right\}$.

The Nevanlinna order function $T_{f}(r, \mathcal{L})$ of $f$ for the line bundle $\mathcal{L}$ is given by:

$$
T_{f}(r, \mathcal{L}):=\int_{r_{0}}^{r} \frac{d t}{t} \int_{B(t)} f^{*} \omega \wedge \psi^{m-1},
$$

where $\omega=\left\{\omega_{\alpha}\right\}=d d^{c} \log \sum_{j=0}^{n}\left(\left|w_{j} / w_{\alpha}\right|^{2}\right)^{d}$ in a neighborhood $U_{\alpha}:=\left\{w_{\alpha} \neq 0\right\}$. We say that a meromorphic mapping $f$ is transcendental if

$$
\lim _{r \rightarrow+\infty} \frac{T_{f}(r, \mathcal{L})}{\log r}=+\infty .
$$

A meromorphic mapping $f$ is rational if and only if $T_{f}(r, \mathcal{L})=O(\log r)(r \rightarrow$ $+\infty)$. The norm of a section $\delta$ is given by

$$
\|\delta\|^{2}:=\frac{\left|\delta_{\alpha}\right|^{2}}{a_{\alpha}}=\frac{|P(w)|^{2}}{\left(\sum_{j=0}^{n}\left|w_{j}\right|^{2}\right)^{d}} .
$$

We may assume $\|\delta\| \leq 1$. The proximity function $m_{f}(r, D)$ of $D$ is defined by

$$
m_{f}(r, D):=\int_{\partial B} \log \frac{1}{\left\|\delta_{f}\right\|} \sigma=\int_{\partial B} \log \frac{\|f\|^{d}}{|P(f)|} \sigma .
$$

The Nevanlinna deficiency $\delta_{f}(D)$ and the Valiron deficiency $\Delta_{f}(D)$ of $D$ for $f$ is defined by

$$
\delta_{f}(D):=\liminf _{r \rightarrow \infty} \frac{m_{f}(r, D)}{T_{f}(r, \mathcal{L})} \text { and } \delta_{f}(D):=\limsup _{r \rightarrow \infty} \frac{m_{f}(r, D)}{T_{f}(r, \mathcal{L})} .
$$

In particular, if $\mathcal{L}$ is the hyperplane bundle $[H]$ and $D$ is a hyperplane $H$ which is given by a vector $\mathbf{a}=\left(a_{0}, \ldots, a_{n}\right) \in \mathbb{C}^{n+1} \backslash\{0\}$, the proximity function $m_{f}(r, H)$ and the counting function $N_{f}(r, H)$ of a hyperplane $H$ in $\mathbb{P}^{n}(\mathbb{C})$ are given by:

$$
m_{f}(r, H):=\int_{\partial B(r)} \log \frac{\|f\|\|\mathbf{a}\|}{|\langle f, \mathbf{a}\rangle|} \sigma \text { and } N_{f}(r, H):=\int_{r_{0}}^{r} \frac{d t}{t} \int_{\left(f^{*} H\right) \cap B(t)} \psi^{m-1}
$$

for some fixed $r_{0}>0$, where $H=\left\{w=\left(w_{0}, \ldots, w_{n}\right) \in \mathbb{C}^{n+1} \backslash\{0\} \mid \sum_{j=0}^{n} a_{j} w_{j}=\right.$ $0\}$ and $f^{*} H$ denotes the pullback of $H$ under $f$. Also, the Nevanlinna order function $T_{f}(r) \equiv T_{f}(r,[H])$ of $f$ for the hyperplane bundle $[H]$ is written as:

$$
T_{f}(r)=\int_{\partial B(r)} \log \left(\sum_{j=0}^{n}\left|f_{j}\right|^{2}\right)^{1 / 2} \sigma+O(1)=\int_{\partial B(r)} \log \sum_{j=0}^{n}\left|f_{j}\right| \sigma+O(1)
$$


by using Stoke's theorem. We write

$$
N(r,(\phi)):=\int_{r_{0}}^{r} \frac{d t}{t} \int_{(\phi) \cap B(t)} \psi^{m-1}
$$

where $(\phi)$ denotes the divisor determined by a meromorphic function $\phi$ on $\mathbb{C}^{m}$.

Let $f: \mathbb{C}^{m} \rightarrow \mathbb{P}^{n}(\mathbb{C})$ be a meromorphic mapping with a reduced representation $\left(f_{0}: \ldots: f_{n}\right)$. Let $\phi: \mathbb{C}^{m} \rightarrow \mathbb{P}^{n}(\mathbb{C})^{*}$ be a meromorphic mapping with a reduced representation $\left(\phi_{0}: \ldots: \phi_{n}\right)$, which is called a moving target for $f$. Then the proximity function $m_{f}(r, \phi)$ and the counting function $N_{f}(r, \phi)$ of a moving target $\phi$ into $\mathbb{P}^{n}(\mathbb{C})^{*}$ are given by:

$$
m_{f}(r, \phi):=\int_{\partial B} \log \frac{\|f\|\|\phi\|}{|\langle f, \phi\rangle|}\left(r e^{i \theta}\right) d \theta \text { and } N_{f}(r, \phi):=\int_{B(r) \cap(A)_{0}} \psi^{m-1}
$$

where $\|f\|^{2}=\sum_{j=0}^{n}\left|f_{j}\right|^{2}$ and $(A)_{0}$ denotes the divisor determined by the zeros of $A:=\langle f, \phi\rangle=\sum_{j=0}^{n} \phi_{j} f_{j}$. The Nevanlinna deficiency $\delta_{f}(\phi)$ and the Valiron deficiency $\Delta_{f}(\phi)$ of a moving target $\phi$ for $f$ are given by:

$$
\delta_{f}(\phi):=\liminf _{r \rightarrow+\infty} \frac{m_{f}(r, \phi)}{T_{f}(r)+T_{\phi}(r)} \text { and } \Delta_{f}(\phi):=\limsup _{r \rightarrow+\infty} \frac{m_{f}(r, \phi)}{T_{f}(r)+T_{\phi}(r)} .
$$

We now define the projective logarithmic capacity of a set in the projective space $\mathbb{P}^{n}(\mathbb{C})$. (see, Molzon-Shiffman-Sibony [7, p. 46]). Let $E$ be a compact subset of $\mathbb{P}^{n}(\mathbb{C})$, and $\mathcal{P}(E)$ denotes the set of probability measures supported on $E$. We set

$$
\begin{aligned}
V_{\mu}(x) & :=\int_{w \in \mathbb{P}^{n}(\mathbb{C})} \log \frac{\|x\|\|w\|}{|\langle x, w\rangle|} d \mu(w) \quad(\mu \in \mathcal{P}(E)) \quad \text { and } \\
V(E) & :=\inf _{\mu \in \mathcal{P}(E)} \sup _{x \in \mathbb{P}^{n}(\mathbb{C})} V_{\mu}(x) .
\end{aligned}
$$

Define the projective logarithmic capacity $C(E)$ of $E$ by

$$
C(E):=\frac{1}{V(E)}
$$

If $V(E)=+\infty$, we say that the set $E$ is of projective logarithmic capacity zero. For an arbitrary subset $K$ of $\mathbb{P}^{n}(\mathbb{C})$, we put

$$
C(K)=\sup _{E \subset K} C(E),
$$

where the supremum is taken over all compact subsets $E$ of $K$. 


\section{2-2. Some Results}

A.Vitter [9] proved the following theorem:

Theorem A (Lemma of the logarithmic derivatives). Let $f=\left(f_{0}: f_{1}\right)$ be a reduced representation of a meromorphic mapping $f: \mathbb{C}^{m} \rightarrow \mathbb{P}^{1}(\mathbb{C})$. Set $F=$ $f_{1} / f_{0}$. Then there exist positive constants $a_{1}, a_{2}, a_{3}$ such that

$$
\int_{\partial B(r)} \log ^{+}\left|F_{z_{j}} / F\right| \sigma \leq a_{1}+a_{2} \log r+a_{3} \log T_{f}(r), \quad(j=1, \ldots, m) . \quad / / .
$$

Here the notation " $A(r) \leq B(r) / /$ " means that the inequality $A(r) \leq B(r)$ holds for $r$ outside a Borel set with finite Lebesgue measure.

Molzon-Shiffman-Sibony proved the following result on the projective logarithmic capacity.

Theorem B [7, p. 47]. Let $\varphi:[0,1] \rightarrow \mathbb{P}^{n}(\mathbb{C})$ be a real smooth nondegenerate arc in $\mathbb{P}^{n}(\mathbb{C})$, and $K$ a compact subset of the interval $[0,1] \subset \mathbb{C}$. Then the projective logarithmic capacity $C(\varphi(K))$ is positive if and only if $K$ has a positive logarithmic capacity in $\mathbb{C}$.

Here "smooth nondegenerate arc $\varphi$ " means that there exists a lift $\tilde{\varphi}:[0,1] \rightarrow$ $\mathbb{C}^{n+1} \backslash\{0\}$ such that the $k$ th derivatives $\left\{\tilde{\varphi}^{(k)}(t)\right\}_{k \geq 0}$ of $\tilde{\varphi}(t)$ spans $\mathbb{C}^{n+1}$ for every $t \in[0,1]$.

Theorem C [4]. Let $f$ be a meromorphic mapping of $\mathbb{C}^{m}$ into $\mathbb{P}^{n}(\mathbb{C})$ such that $\lim _{r \rightarrow+\infty} T_{f}(r)=+\infty$. Then there exist a sequence $r_{1}<r_{2}<\ldots<r_{n} \rightarrow+\infty$ and sets $E_{n}: E_{n+1} \subset E_{n}(n=1,2, \ldots)$ in $\mathbb{P}^{n}(\mathbb{C})^{*}$ with $V\left(E_{n}\right) \geq 2 \log T_{f}\left(r_{n}\right)$ such that, if $H$ does not belong to $E_{n}$, then

$$
m_{f}(r, H) \leq 4 \sqrt{T_{f}(r)} \log T_{f}(r)
$$

for $r>r_{n}$. Hence

$$
\lim _{r \rightarrow+\infty} \frac{m_{f}(r, H)}{T_{f}(r)}=0
$$

outside a set $E \subset \mathbb{P}^{n}(\mathbb{C})^{*}$ of projective logarithmic capacity zero. Here $\mathbb{P}^{n}(\mathbb{C})^{*}$ denotes the dual projective space of $\mathbb{P}^{n}(\mathbb{C})$.

Theorem D [1]. Set $\Lambda(r):=\int_{r_{0}}^{r} \psi(t) / d t t$, where $\psi(r)$ is nonnegative, nondecreasing and unbounded. If $\Lambda(r)<r^{K}$ for some $K>0$ and all sufficiently large $r$, then there exists an entire function $g(z)$ of finite order such that $T_{g}(r) \sim \Lambda(r)(r \rightarrow \infty)$. 


\section{Elimination of Defects of Meromorphic Mappings}

\section{3-1. Elimination of deficient hyperplanes of a meromorphic mapping}

For a transcendental meromorphic mapping $g$ of $\mathbb{C}^{m}$ into $\mathbb{P}^{n}(\mathbb{C})$, we can eliminate all deficient hyperplanes by a small deformation of $g$.

Lemma 1 [4]. There are monomials $\zeta_{1}, \ldots, \zeta_{n}$ in $z_{1}, \ldots, z_{m}$ such that any $n$ derivatives in $\left\{D^{\alpha} \zeta:=\left(D^{\alpha} \zeta_{1}, \ldots, D^{\alpha} \zeta_{n}\right)|| \alpha \mid \leq n+1\right\}$ are linearly independent over the field $M$ of meromorphic functions on $\mathbb{C}^{m}$, where $\alpha=\left(\alpha_{1}, \ldots, \alpha_{m}\right) \in \mathbb{Z}_{\geq 0}$ is a multi-index.

Lemma 2 [4]. Let $h=\left(h_{0}: h_{1}: \cdots: h_{n}\right)$ be a reduced representation of a meromorphic mapping of $\mathbb{C}^{m}$ into $\mathbb{P}^{n}(\mathbb{C})$ and $\zeta_{1}, \ldots, \zeta_{n}$ linearly independent monomiales in $z_{1}, \ldots, z_{m}$ as in Lemma 1 . Then there exists $\left(\tilde{a}_{1}, \ldots, \tilde{a}_{n}\right)$ such that $\tilde{a}_{j}=\alpha^{k_{j}}(j=1, \ldots, n)$ with $k_{1}=1, k_{m}=\sum_{l=1}^{m-1} k_{l}+1(m=2,3, \ldots, n)(\alpha \in \mathbb{C})$, and

$$
f:=\left(h_{0}: h_{1}+\tilde{a}_{1} \zeta_{1} h_{0}: h_{2}+\tilde{a}_{2} \zeta_{2} h_{0}: \cdots: h_{n}+\tilde{a}_{n} \zeta_{n} h_{0}\right)
$$

is a reduced representation of a linearly nondegenerate meromorphic mapping of $\mathbb{C}^{m}$ into $\mathbb{P}^{n}(\mathbb{C})$.

Lemma 3 [4]. Let $f=\left(f_{0}: \cdots: f_{n}\right)$ and $h=\left(h_{0}: \cdots, h_{n}\right)$ be as in Lemma 2. Then we have

$$
\left|T_{f}(r)-T_{h}(r)\right| \leq O(\log r) \quad(r \rightarrow \infty)
$$

Lemma 4 [4]. The set of vectors

$$
\mathcal{A}:=\left\{\left(1, a_{1}, \ldots, \prod_{k=1}^{n} a_{k}\right) \mid a_{j} \in \mathbb{C}\right\}
$$

is of positive projective logarithmic capacity in $\mathbb{P}^{N}(\mathbb{C})$, where $N=2^{n}-1$.

Theorem 1 [4]. Let $g: \mathbb{C}^{m} \rightarrow \mathbb{P}^{n}(\mathbb{C})$ be a given transcendental meromorphic mapping. Then there exists a regular matrix $L=\left(l_{i j}\right)_{0 \leq i, j \leq n}$ of the form $l_{i, j}=$ $c_{i j} \zeta_{i}+d_{i j},\left(c_{i j}, d_{i j} \in \mathbb{C}: 0 \leq i, j \leq n\right)$, such that $\operatorname{det} L \neq 0$ and $f:=L \cdot g: \mathbb{C}^{m} \rightarrow$ $\mathbb{P}^{n}(\mathbb{C})$ is a meromorphic mapping without Nevanlinna deficient hyperplanes, where $\zeta_{1}, \ldots, \zeta_{n}$ are some monomials in $z_{1}, \ldots, z_{m}$ which are linearly independent over $\mathbb{C}$.

Here the mapping $f:=L \cdot g: \mathbb{C}^{m} \rightarrow \mathbb{P}^{n}(C)$ means a product of the matrix $L=\left(l_{i j}\right)$ and a vector of a reduced representation $\tilde{g}={ }^{t}\left(g_{0}: \ldots: g_{n}\right)$ of $g$ which does not depend on a choice of $\tilde{g}$, and also a Nevanlinna deficient hyperplane $H$ for $f$ means a hyperplane with $\delta_{f}(H)>0$. 
Remark 1. For the mappings as in Theorem 1, the inequality $\left|T_{f}(r)-T_{g}(r)\right| \leq$ $O(\log r)(r \rightarrow+\infty)$ holds, and also the mapping $g$ may be linearly degenarate or of infinite order.

Remark 2. A rational mapping $g$ always has a Nevanlinna deficient hyperplane if $m=1$ or there is a regular linear change $L_{0}$ such that $L_{0} \cdot g$ has a reduced representation which consists of polynomials including different degrees. But otherwise $g$ does not have Nevanlinna deficient hyperplanes.

Remark 3. If $g$ is of finite order, we can replace "Nevanlinna deficiency" by "Valiron deficiency" in the conclusion of Theorem 1.

Remark 4. If $m=1$, we can take $\zeta_{k}=z^{k} \quad(k=1, \ldots, n)$.

Outline of the proof of Theorem 1 (see [4]).

1st step. There is a regular linear change $L_{1}$ of $\mathbb{P}^{n}(\mathbb{C})$ such that

$$
h:=L_{1} \cdot g=\left(h_{0}: \cdots: h_{n}\right): \mathbb{C}^{m} \longrightarrow \mathbb{P}^{n}(\mathbb{C})
$$

and a reduced representation of the meromorphic mapping $h$ which satisfies

$$
N\left(r,\left(h_{j}\right)\right)=(1-o(1)) T_{h}(r), \quad(r \rightarrow+\infty), \quad(j=0,1, . ., n) .
$$

2nd step. Using Theorems B and C, and Lemmas 1 and 2, there are $f=\left(h_{0}\right.$ : $\left.h_{1}+a_{1} \zeta_{1} h_{0}: \ldots: h_{n}+a_{n} \zeta_{n} h_{0}\right)$ and multi-indices $\beta^{0}, \ldots, \beta^{n}$ such that $f$ is linearly nondegenerate and its generalized Wronskian satisfies $\mathbf{W}_{\beta}:=\mathbf{W}_{\beta^{0}, \ldots, \beta^{n}}(f) \not \equiv 0$. Note that there are many such $\left\{a_{1}, \ldots, a_{n}\right\}$. Then it can be written as

$$
\mathbf{W}_{\beta}=h_{0}^{n+1}\left(W_{0}+a_{1} W_{1}+\cdots+\prod_{i=1}^{n} a_{i} W_{N}\right) \not \equiv 0,
$$

where $W_{k}$ is a generalized Wronskian of some of $1, h_{1} / h_{0}, a_{1} \zeta_{1}, \ldots, h_{n} / h_{0}, a_{n} \zeta_{n}$ $\left(0 \leq k \leq N=2^{n}-1\right)$.

3rd step. Consider the auxiliary meromorphic mapping $F$ of the form

$$
F:=\left(W_{0} / d: W_{1} / d: \cdots: W_{N} / d\right): \mathbb{C}^{m} \longrightarrow \mathbb{P}^{N}(\mathbb{C}),
$$

where $d=d(z)$ is a meromorphic function which consists of common factors among $W_{0}, \ldots, W_{N}$ such that $W_{0} / d, \ldots, W_{N} / d$ are holomorphic functions without common factors up to unit. Then we observe that the meromorphic mapping $F$ is not constant. Therefore, there exists an $\mathbf{a}_{0}=\left(1, \tilde{a}_{1}, \ldots, \tilde{a}_{n}, \tilde{a}_{1} \tilde{a}_{2}, \ldots, \prod_{j=1}^{n} \tilde{a}_{j}\right)$ such that

$$
\limsup _{r \rightarrow \infty} \frac{m_{F}\left(r, H_{\mathbf{a}_{0}}\right)}{T_{F}(r)}=0
$$


since the set of Valiron deficient hyperplanes of a nonconstant meromorphic mapping is of projective logarithmic capacity zero in $\mathbb{P}^{N}(\mathbb{C})^{*}$.

4th step. Consider the meromorphic mapping given by the following reduced representation by using the vector $\mathbf{a}_{0}$ in the 3 rd step:

$$
f:=L_{2} \cdot h=\left(f_{0}: \cdots: f_{n}\right): \mathbb{C}^{m} \longrightarrow \mathbb{P}^{n}(\mathbb{C}),
$$

where

$$
L_{2}=\left(\begin{array}{cccc}
1 & 0 & \cdots & 0 \\
\tilde{a}_{1} \zeta_{1} & 1 & \cdots & 0 \\
\tilde{a}_{2} \zeta_{2} & 0 & \cdots & 0 \\
& \cdots & \cdots & \\
\tilde{a}_{n} \zeta_{n} & 0 & \cdots & 1
\end{array}\right), \quad\left(\operatorname{det} L_{2}=1 \neq 0\right)
$$

Hence $f_{0}=h_{0}$ and $f_{k}=h_{k}+\tilde{a}_{k} \zeta_{k} h_{0}(k=1, \ldots, n)$. Then we observe that

$$
T_{f}(r)=T_{g}(r)+O(\log r)=(1+o(1)) T_{g}(r),(r \rightarrow+\infty),
$$

if $\mathrm{g}$ is not rational.

Claim 1. Let $F$ and $f$ be as above. Then there exists a positive constant $K$ such that

$$
T_{F}(r) \leq K T_{f}(r)
$$

5th step. Take an arbitrary vector $\mathbf{b}=\left(b_{0}, \ldots, b_{n}\right) \in \mathbb{C}^{n+1} \backslash\{0\}$, which determines the hyperplane $H=\left\{w \in \mathbb{C}^{n+1} \backslash\{0\} \mid\langle w, \mathbf{b}\rangle=0\right\}$ in $\mathbb{P}^{n}(\mathbb{C})$. We may assume that $b_{n} \neq 0$. Then $f_{0}, f_{1}, \ldots, f_{n-1}, A=\langle f, \mathbf{b}\rangle$ are linearly independent over $\mathbb{C}$. Thus we have

$$
\begin{aligned}
& m_{f}\left(r, H_{\mathbf{b}}\right)=\int_{\partial B(r)} \log \frac{\|f\|}{|A|} \sigma \\
= & \int_{\partial B(r)} \log \frac{\left|W_{\beta^{0}, \ldots, \beta^{n}}\left(f_{0}, . ., f_{n}\right)\right|}{|A|\left|f_{0}\right| \cdots\left|f_{n-1}\right|} \sigma+\int_{\partial B(r)} \log \frac{\|f\|\left|f_{0}\right| \cdots\left|f_{n-1}\right|}{\left|W_{\beta^{0}, . ., \beta^{n}}\left(f_{0}, . ., f_{n}\right)\right|} \sigma \\
\leq & \int_{\partial B(r)} \log \frac{\left|b_{n}^{-1}\right|\left|W_{\beta^{0}, ., \beta^{n}}\left(f_{0}, . ., f_{n-1}, A\right)\right|}{|A|\left|f_{0}\right| \cdots\left|f_{n-1}\right|} \sigma+\int_{\partial B(r)} \log \frac{\|f\|^{n+1}}{\left|f_{0}\right|^{n+1}} \sigma \\
& +\int_{\partial B(r)} \log \frac{1}{\left|W_{0}+a_{1} W_{1}+\cdots+\prod_{j=1}^{n} a_{j} W_{N}\right|} \sigma+O(1), \\
\leq & o\left(T_{f}(r)\right)+(n+1) m_{f}\left(r, H_{(1,0, . ., 0)}\right) \\
& +\int_{\partial B(r)} \log \frac{\left(\left|W_{0}\right|+\left|W_{1}\right|+\cdots+\left|W_{N}\right|\right)(1 /|d|)}{\left|W_{0}+a_{1} W_{1}+\cdots+\prod_{j=1}^{n} a_{j} W_{N}\right|(1 /|d|)} \sigma+O(1)
\end{aligned}
$$




$$
=o\left(T_{f}(r)\right)+\int_{\partial B(r)} \log \frac{\|F\|}{\left|\left\langle F, \mathbf{a}_{0}\right\rangle\right|} \sigma=o\left(T_{f}(r)\right)+o\left(T_{F}(r)\right)=o\left(T_{f}(r)\right) / / .
$$

Therefore, we obtain

$$
\delta_{f}\left(H_{\mathbf{b}}\right)=\liminf _{r \rightarrow+\infty} \frac{m_{f}\left(r, H_{\mathbf{b}}\right)}{T_{f}(r)}=0,
$$

that is, $\delta_{f}(H)=0$ for any $H \in \mathbb{P}^{n}(\mathbb{C})^{*}$. This proves Theorem 1 .

Note that we can take the norm $\|\tilde{\boldsymbol{\alpha}}\|$ of a vector $\tilde{\boldsymbol{\alpha}}:=\left(\tilde{a}_{1}, \ldots, \tilde{a}_{n}\right)$ as small as possible in the proof of Theorem 1 .

Problem. Is the conclusion of Theorem 1 true if "Nevanlinna deficiency" is replaced by "Valiron deficiency"?

\section{3-2. Elimination of defect hypersurfaces of a holomorphic mapping of $\mathbb{C}$ into} $\mathbb{P}^{n}(\mathbb{C})$

We shall discuss an elimination theorem on defects of hypersurfaces.

Theorem 2 [5]. Let $g$ be a given transcendental holomorphic mapping of $\mathbb{C}$ into $\mathbb{P}^{n}(\mathbb{C})$, and $d \in \mathbb{N}$ be given. Then there exists a regular matrix $L=\left(l_{i j}\right)$ of the form $l_{i j}=c_{i j} z^{m_{j}}+d_{i j},\left(c_{i j}, d_{i j} \in \mathbb{C}\right),|L| \neq 0$ and $f:=L \cdot g: \mathbb{C} \rightarrow \mathbb{P}^{n}(\mathbb{C})$ is a holomorphic mapping without Nevanlinna deficient hypersurfaces of degree $\leq d$, where $m_{j}(j=1, \ldots, n)$ are some integers such that $m_{1}<d m_{1}<m_{2}<\cdots<$ $d m_{n-1}<m_{n}$.

Outline of the proof of Theorem 2. There is a regular linear change $L_{1}$ such that the holomorphic mapping $h:=L_{1} \cdot g=\left(h_{0}: \cdots: h_{n}\right): \mathbb{C} \rightarrow \mathbb{P}^{n}(\mathbb{C})$ satisfies $N\left(r,\left(h_{j}\right)\right)=(1-o(1)) T_{h}(r),(r \rightarrow \infty),(j=0, \ldots, n)$. Consider the Veronese embedding $v_{d}: \mathbb{P}^{n}(\mathbb{C}) \rightarrow \mathbb{P}^{s}(\mathbb{C})$, which is defined by homogeneous monomials of degree $d$ in $\left(w_{0}: \cdots: w_{n}\right) \in \mathbb{P}^{n}(\mathbb{C})$. Let $\tilde{h}=\left(\tilde{h}_{0}: \cdots: \tilde{h}_{n}\right):=\left(h_{0}:\right.$ $\left.h_{1}+a_{1} z^{m_{1}} h_{0}: \cdots: h_{n}+a_{n} z^{m_{n}} h_{0}\right)$. Consider the composed mapping $\hat{f}:=v_{d} \circ \tilde{h}=$ $\left(\hat{f}_{0}: \cdots: \hat{f}_{s}\right)=\left(\tilde{h}_{0}^{d}: \tilde{h}_{0}^{d-1} \tilde{h}_{1}: \cdots: \tilde{h}_{0} \tilde{h}_{1}^{d-1}: \tilde{h}_{1}^{d}: \tilde{h}_{0}^{d-1} \tilde{h}_{2}: \cdots: \tilde{h}_{n}^{d-1} \tilde{h}_{n-1}: \tilde{h}_{n}^{d}\right)$. Here $s=(n+d) ! / d ! n !-1$. We can prove the following Lemma 5 using a similar method for the proof of Theorem 1 .

Lemma 5 [5]. There is a vector $\left(a_{1}, \ldots, a_{n}\right) \in \mathbb{C}^{n} \backslash\{0\}$ such that $\hat{f}$ is linearly nondegenerate.

Set

$$
\begin{aligned}
\mathbf{W} & =\mathbf{W}\left(\hat{f}_{0}, \ldots, \hat{f}_{s}\right)=\mathbf{W}\left(\tilde{h}_{0}^{d}, \tilde{h}_{0}^{d-1} \tilde{h}_{1}, \tilde{h}_{0}^{d-2} \tilde{h}_{1}^{2}, \ldots \ldots, \tilde{h}_{n}^{d}\right) \\
& =\tilde{h}_{0}^{d(s+1)} \mathbf{W}\left(1, \tilde{H}_{1}, \tilde{H}_{1}^{2}, \ldots \ldots, \tilde{H}_{1}^{k_{1}} \tilde{H}_{2}^{k_{2}} \cdots \tilde{H}_{n}^{k_{n}}, \ldots \ldots, \tilde{H}_{s}^{d}\right) \\
& =\tilde{h}_{0}^{d(s+1)}\left(\mathbf{W}_{0}+a_{1} \mathbf{W}_{1}+\cdots+\prod_{k=1}^{n} a_{k}^{d} \mathbf{W}_{N}\right),\left(\tilde{H}_{j}=H_{j}+a_{j} z^{m_{j}}\right)
\end{aligned}
$$


where $\mathbf{W}_{j}(j=0, \ldots, N)$ are some sums of Wronskians. Consider the auxiliary holomorphic mapping:

$$
F:=\left(\mathbf{W}_{0} / d(z): \mathbf{W}_{1} / d(z): \cdots: \mathbf{W}_{N} / d(z)\right): \mathbb{C} \longrightarrow \mathbb{P}^{N}(\mathbb{C}) .
$$

Here $d=d(z)$ is a holomorphic function such that $\mathbf{W}_{0} / d, \ldots, \mathbf{W}_{N} / d$ are holomorphic functions without common zeros. Then there is a vector

$$
\mathbf{a} \in \mathcal{A}:=\left\{\left(1, a_{1}, \ldots, \prod_{k=1}^{n} a_{k}^{d}\right) \mid a_{j} \in \mathbb{C}\right\}
$$

such that $m_{F}\left(r, H_{\mathbf{a}}\right)=o\left(T_{F}(r)\right),(r \rightarrow \infty)$, since $\mathcal{A}$ has a positive projective logarithmic capacity by Theorem B. Consider the holomorphic mapping given by the following reduced representation which is determined by the vector $\left(a_{1}, \ldots, a_{n}\right)$ corresponding to above a:

$$
f:=L_{2} \cdot h: \mathbb{C} \longrightarrow \mathbb{P}^{n}(\mathbb{C}),
$$

where $L_{2}=\left(s_{i j}\right)$ and $s_{i j}=1(i=j), s_{i j}=a_{i} z^{m_{i}}(j=1, i \neq 1), s_{i j}=0$ (otherwise). Then $\operatorname{det}\left(s_{i j}\right) \neq 0$.

Claim 2. There is a positive constant $K$ such that $T_{F}(r) \leq K T_{h}(r)$, and also $(1+o(1)) T_{f}(r)=T_{g}(r)=(1+o(1)) T_{h}(r),(r \rightarrow \infty)$, hold by a similar method in Section 3-1.

Now we take an arbitrary hypersurface $D=D_{\mathbf{b}}$ in $\mathbb{P}^{n}(\mathbb{C})$ which is determined by a homogeneous polymonial:

$$
\left.P(w):=b_{0} w_{0}^{d}+b_{1} w_{0}^{d-1} w_{1}+\cdots+b_{k} w_{0}^{j_{0}} w_{1}^{j_{1}} \cdots w_{n}^{j_{n}}+\cdots+b_{s} w_{n}^{d}=0\right\},
$$

$w=\left(w_{0}, \ldots, w_{n}\right) \in \mathbb{C}^{n+1} \backslash\{0\}$. Then $D$ corresponds to the vector $\mathbf{b}=\left(b_{0}, \ldots, b_{s}\right)$. We may assume that $b_{s} \neq 0$. We set $\tilde{f}:=v_{d} \circ f$. Consider the function

$$
A_{\mathbf{b}}=\sum_{k=0}^{s} b_{k} f_{0}^{j_{0}^{k}} \cdots f_{n}^{j_{n}^{k}}
$$

where $J_{k}:=\left(j_{0}^{k}, \ldots, j_{n}^{k}\right)$ with $\left|J_{k}\right|:=j_{0}^{k}+\cdots+j_{n}^{k}=d$. Then $\hat{f}_{0}, \ldots, \hat{f}_{s-1}, A_{\mathbf{b}}$ are linearly independent over $\mathbb{C}$, since $\hat{f}:=\left(\hat{f}_{0}: \cdots: \hat{f}_{s}\right)$ is linearly nondegenerate. Then, using Theorem A, Claim 2 and the similar method to the proof of Theorem 1 , we obtain

$$
m_{f}\left(r, D_{\mathbf{b}}\right)=\int_{\partial B(r)} \log \frac{\|f\|^{d}}{\left|A_{\mathbf{b}}\right|} \sigma=o\left(T_{f}(r)\right) .
$$


Therefore, we obtain

$$
\delta_{f}\left(D_{\mathbf{b}}\right)=\liminf _{r \rightarrow \infty} \frac{m_{f}\left(r, D_{\mathbf{b}}\right)}{d T_{f}(r)}=0 .
$$

In case where hypersurfaces of degree $\leq d$, for each $d^{\prime}(\leq d)$, we can take a vector $\mathbf{a}=\mathbf{a}_{d^{\prime}}$ in a subset of $\mathcal{A}$ of positive projective logarithmic capacity. Hence we can take a common vector $\mathbf{a} \in \mathcal{A}$ for each $d^{\prime}$. This proves Theorem 2 .

Note that Theorem 2 can be extended to the case where meromorphic mappings of $\mathbb{C}^{m}$ into $\mathbb{P}^{n}(\mathbb{C})$ by using the similar method to Section 3-1.

\section{3-3. Elimination of defects of holomorphic curves for rational moving targets}

For a transcendental holomorphic curve $f$ of $\mathbb{C}$ into $\mathbb{P}^{n}(\mathbb{C})$, we can eliminate all defects of rational moving targets by a small deformation of $f$.

Theorem 3 [6]. Let $f: \mathbb{C} \rightarrow \mathbb{P}^{n}(\mathbb{C})$ be a given transcendental holomorphic curve. Then there exists a regular matrix

$$
L=\left(l_{i j}\right)_{0 \leq i, j \leq n} \text { of the form } l_{i, j}={ }_{i j} g_{j}+d_{i j},\left(c_{i j}, d_{i j} \in \mathbb{C}: 0 \leq i, j \leq n\right),
$$

such that $\operatorname{det} L \neq 0$ and $\tilde{f}=L \cdot f: \mathbb{C} \rightarrow \mathbb{P}^{n}(\mathbb{C})$ is a holomorphic curve without Nevanlinna defects of rational moving targets and satisfies

$$
\left|T_{f}(r)-T_{\tilde{f}}(r)\right|=o\left(T_{f}(r)\right), \quad(r \rightarrow \infty),
$$

where $g_{j}(j=1, \ldots, n)$ are some transcendental entire functions satisfying $T_{g_{j}}(r)=$ $o\left(T_{g_{j+1}}(r)\right),(j=1, \ldots, n-1)$, and $T_{g_{n}}=o\left(T_{f}(r)\right)(r \rightarrow \infty)$.

Note that we cannot replace transcendental entire functions $g_{j}$ by any rational functions.

Problem: Can we extend Theorem 3 to the case of several complex variables?

Outline of the proof of Theorem 3. Let $h$ be a transcendental holomorphic curve and $\left(h_{0}, \ldots, h_{n}\right)$ its reduced representation. Then there are indices $i, j$ such that $h_{j} / h_{i}$ is transcendental, say $i=0, j=n$. By Theorem $\mathrm{D}$, there are $n$ transcendental entire functions $g_{1}, \ldots, g_{n}$ on $\mathbb{C}$ such that $T_{g_{j}}(r)=o\left(T_{g_{j+1}}\right),(j=1, \ldots, n-1)$ and $T_{g_{n}}(r)=o\left(T_{f}(r)\right)$ as $r \rightarrow \infty$. Then $g_{1}, \ldots, g_{n}$ are linearly independent over $\mathbb{C}$. There is a regular linear change $L_{1}$ such that

$$
h=L_{1} \cdot f=\left(h_{0}: \cdots: h_{n}\right): \mathbb{C} \longrightarrow \mathbb{P}^{n}(\mathbb{C}),
$$

and a reduced representation of the holomorphic curve $h$ satisfying

$$
N\left(r, 0, h_{j}\right) \sim T_{h}(r), \quad(r \rightarrow+\infty), \quad(j=0, \ldots, n) .
$$


We put $\bar{h}_{k}=h_{k}+a_{k} g_{k} h_{0}(k=1, \ldots, n)$ and $\bar{h}_{0}=h_{0}$. Consider the reduced representation of a holomorphic curve

$$
\bar{h}:=\left(\bar{h}_{0}: \bar{h}_{1}: \cdots: \bar{h}_{n}\right): \mathbb{C} \longrightarrow \mathbb{P}^{n}(\mathbb{C}) .
$$

Then there exist complex numbers $a_{1}, \ldots, a_{n}$ such that

$$
\left\{\bar{h}_{0}, z \bar{h}_{0}, \ldots, z^{m} \bar{h}_{0}, \bar{h}_{1}, \ldots, z^{m} \bar{h}_{1}, \ldots \ldots, \bar{h}_{n}, \ldots, z^{m} \bar{h}_{n}\right\}
$$

is linearly independent over $\mathbb{C}$, as in previous theorems.

We now consider the Wronskian

$$
\mathbf{W}:=W\left(\bar{h}_{0}, z \bar{h}_{0}, \ldots, z^{m} \bar{h}_{0}, \bar{h}_{1}, \ldots, z^{m} \bar{h}_{1}, \ldots \ldots, \bar{h}_{n}, \ldots, z^{m} \bar{h}_{n}\right),
$$

and we write it as

$$
\begin{aligned}
\mathbf{W}:= & W_{0}\left(h_{0}, z h_{0}, \ldots, z^{m} h_{0}, h_{1}, \ldots, z^{m} h_{1}, \ldots, h_{n}, \ldots, z^{m} h_{n}\right) \\
& +a_{1}\left(W_{11}+\cdots+W_{1 s_{1}}\right)+\cdots+a_{n}\left(W_{n 1}+\cdots+W_{n s_{n}}\right) \\
& +a_{1}^{2}\left(W_{1^{2} 1}+\cdots+W_{1^{2} s_{1}^{2}}\right)+\cdots+a_{1}^{m+1}\left(W_{1^{m+1} 1}+\cdots+W_{1^{m+1} s_{1}^{m+1}}\right) \\
& +a_{1} a_{2}\left(W_{1^{12} 1}+\cdots+W_{1^{12} s_{12}}\right)+\cdots \\
& +\prod_{j=1}^{n} a_{j}^{m+1} W_{N}\left(1, \ldots, z^{m}, g_{1}, \ldots, z^{m} g_{1}, \ldots, g_{n}, \ldots, z^{m} g_{n}\right) \cdot h_{0}^{(m+1)(n+1)}
\end{aligned}
$$

We now rewrite it in an inhomogeneous form as

$$
\mathbf{W}=h_{0}^{(m+1)(n+1)}\left\{\mathbf{W}_{0}+a_{1} \mathbf{W}_{1}+\cdots+\prod_{j=1}^{n} a_{j}^{m+1} \mathbf{W}_{N}\right\},
$$

where $\mathbf{W}_{k}(k=0, \ldots, N)$ are sums of some Wronskian determinants, and $N=$ $(m+2)^{n}-1$. For any fixed $m \in \mathbb{N}$, we consider an auxiliary holomorphic curve of the form

$$
F_{m}:=\left(\mathbf{W}_{0} / d: \mathbf{W}_{1} / d: \cdots: \mathbf{W}_{N} / d\right): \mathbb{C} \longrightarrow \mathbb{P}^{N}(\mathbb{C}),
$$

where $d=d(z)$ is a meromorphic function whose zeros and poles consist of common factors among $\mathbf{W}_{0}, \ldots, \mathbf{W}_{N}$. Then $F_{m}$ is a reduced representation of nonconstant holomorphic curve in $\mathbb{P}^{N}(\mathbb{C})$.

Lemma 6 [cf. 6]. Let

$$
\begin{aligned}
\mathcal{A}:= & \left\{\left(1, a_{1}, \ldots, a_{1}^{m+1}, a_{2}, \ldots, a_{1}^{i_{1}} a_{2}^{i_{2}} \cdots a_{n}^{i_{n}}, \ldots ., \prod_{j=1}^{n} a_{j}^{m+1}\right) \mid a_{j} \in \mathbb{C},\right. \\
& \left.0 \leq i_{1}, \ldots, i_{n} \leq m+1\right\} .
\end{aligned}
$$


Then there is a vector $\mathbf{a}=\left(1, a_{1}, \ldots, \prod_{j=1}^{n} a_{j}^{m+1}\right)$ such that

$$
\limsup _{r \rightarrow \infty} \frac{m_{F_{m}}\left(r, H_{\mathbf{a}}\right)}{T_{F_{m}}(r)}=0 .
$$

This holds for any positive integer $m$, because a countable union of sets of projective logarithmic capacity zero is of projective logarithmic capacity zero. Here

$$
\begin{aligned}
H_{\mathbf{a}}= & \left\{\zeta=\left(\zeta_{0}, \ldots, \zeta_{N}\right) \mid\langle\zeta, \mathbf{a}\rangle=0\right\} \quad \text { and } \\
& \langle F, \mathbf{a}\rangle=\left\{\mathbf{W}_{0}+a_{1} \mathbf{W}_{1}+\cdots+\prod_{j=1}^{n} a_{j}^{m+1} \mathbf{W}_{N}\right\} / d .
\end{aligned}
$$

Lemma 7 [6]. Let $F_{m}$ and $h$ be as above. Then there exists a positive constant $K$ such that

$$
T_{F_{m}}(r) \leq K T_{h}(r) .
$$

For this $\left(a_{1}, . ., a_{n}\right)$, we consider the holomorphic curve given by the following reduced representation:

$$
\tilde{f}:=L_{2} \cdot h \equiv\left(\tilde{f}_{0}, \ldots, \tilde{f}_{n}\right): \mathbb{C} \longrightarrow \mathbb{P}^{n}(\mathbb{C}),
$$

where $L_{2}:=\left(s_{i j}\right)$ and $s_{i j}=1(i=j), s_{i j}=a_{i} g_{i}(j=1, i \neq 1), s_{i j}=0$ (otherwise). Hence $\tilde{f}_{0}=h_{0}, \tilde{f}_{k}=h_{k}+a_{k} g_{k} h_{0}(k=1, \ldots, n)$, and $\operatorname{det}\left(s_{i j}\right) \neq 0$. Then we see

$$
T_{\tilde{f}}(r)=T_{f}(r)+o\left(T_{\bar{h}}(r)\right)=(1+o(1)) T_{f}(r) \quad(r \rightarrow+\infty) .
$$

Now we take a given integer $m$ and an arbitrary rational target $\phi$ of degree $m$ :

$$
\phi=\left(\phi_{0}(z), \ldots, \phi_{n}(z)\right): \mathbb{C} \longrightarrow \mathbb{P}^{n}(\mathbb{C})^{*} .
$$

Then we can choose a reduced representation of $\phi$ such that each $\phi_{j}$ is a polynomial of degree $\leq m$ and some $\phi_{i_{0}}$ is of degree $m$. Put $A_{m}:=\langle\tilde{f}, \phi\rangle=$ $\sum_{k=0}^{n} \phi_{k} \tilde{f}_{k}$. We may assume that $\phi_{n}=b_{0}^{n}+b_{1}^{n} z+\cdots+b_{m_{\tilde{f}}}^{n} z^{m} \not \equiv 0$. We note that $\tilde{f}_{0}, z f_{0}, \ldots, z^{m} \tilde{f}_{0}, \ldots \ldots, \tilde{f}_{n-1}, z \tilde{f}_{n-1}, \ldots, z^{m} \tilde{f}_{n-1}, \tilde{f}_{n}, \ldots, z^{m-1} \tilde{f}_{n}, A_{m}$ are linearly independent over $\mathbb{C}$. Thus we have

$$
m_{\tilde{f}}(r, \phi)=\frac{1}{2 \pi} \int_{0}^{2 \pi} \log \frac{\|\tilde{f}\|}{\left|A_{m}\right|} d \theta=o\left(T_{\tilde{f}}(r)\right), / /
$$

by Lemma 7 and using a similar method to the proof of Theorem 1 . Here $s=$ $m(m+1)(n+1) / 2$. Therefore, we obtain

$$
\delta_{\tilde{f}}(\phi)=\liminf _{r \rightarrow+\infty} \frac{m_{\tilde{f}}(r, \phi)}{T_{\tilde{f}}(r)}=0 .
$$

We note that $\hat{f}:=L_{1}^{-1} \cdot \tilde{f}$ is also a small deformation of $f$. 


\section{Space of Meromorphic Mappings Into $\mathbb{P}^{n}(\mathbb{C})$}

We shall introduce a distance on the space of meromorphic mappings into $\mathbb{P}^{n}(\mathbb{C})$.

For points $\mathbf{a}=\left(a_{0}: \ldots: a_{n}\right)$ and $\mathbf{b}=\left(b_{0}: \ldots: b_{n}\right)$ in $\mathbb{P}^{n}(\mathbb{C})$, we define the distance $d(\mathbf{a}, \mathbf{b})$ by

$$
d_{1}(\mathbf{a}, \mathbf{b}):=\inf _{\theta}\left\|\frac{\mathbf{a}}{\|\mathbf{a}\|}-e^{i \theta} \frac{\mathbf{b}}{\|\mathbf{a}\|}\right\| .
$$

Then $d_{1}(\mathbf{a}, \mathbf{b})$ satisfies the condition of a distance. Let $f=\left(f_{0}: \ldots: f_{n}\right)$ and let $g=\left(g_{0}: \ldots: g_{n}\right)$ be reduced representations of meromorphic mappings of $\mathbb{C}^{m}$ into $\mathbb{P}^{n}(\mathbb{C})$. Then the distance $d(f(z), g(z))$ at $z \in \mathbb{C}^{m}$ is given by

$$
d_{1}(f(z), g(z))=\inf _{\theta}\left\|\frac{f(z)}{\|f(z)\|}-e^{i \theta} \frac{g(z)}{\|g(z)\|}\right\| \leq 2 .
$$

Define the distance $d(f, g)$ by $d(f, g):=d_{1}(f, g)+d_{2}(f, g)$. Here

$$
d_{1}(f, g):=\sum_{n=1}^{\infty} \frac{1}{2^{n+1}} \int_{n}^{n+1} d t \int_{\partial B(t)} d_{1}(f(z), g(z)) \sigma \leq 1,
$$

which is a distance but does not distinguish rational and transcendental mappings, and

$$
\begin{aligned}
d_{2}(f, g):= & \liminf _{\alpha \rightarrow+1} \limsup _{r \rightarrow \infty}\left\{\left|\frac{T_{f}(r)}{(\log r)^{1 / 2}+T_{f}(r)}-\frac{T_{g}(r)}{(\log r)^{1 / 2}+T_{g}(r)}\right|\right. \\
& +\left|\frac{T_{f}(r)}{(\log r)^{\alpha}+T_{f}(r)}-\frac{T_{g}(r)}{(\log r)^{\alpha}+T_{g}(r)}\right| \\
& \left.+\sum_{n=1}^{\infty}\left|\frac{T_{f}(r)}{r^{n}+T_{f}(r)}-\frac{T_{g}(r)}{r^{n}+T_{g}(r)}\right|\right\},
\end{aligned}
$$

which is a pseudodistance and distinguishes rational and transcendental mappings. Then $d(f, g)$ satisfies the distance conditions on the space of meromorphic mappings into $\mathbb{P}^{n}(\mathbb{C})$. Here $\partial B(r)$ denotes the boundary of a ball of radius $r$ and $\sigma$ denotes the normalized surface element as $\int_{\partial B(r)} \sigma=1$ on $\partial B(r)$.

Note that if $f$ is constant, then $0 \leq d_{1}(f, O)<1$ and $d_{2}(f, O)=0$. Hence $0 \leq d(f, O)<1$. If $f$ is rational, then $0 \leq d_{1}(f, O)<1$ and $d_{2}(f, O)=1$. Hence $1 \leq d(f, O)<2$. If $f$ is transcendental, then $0 \leq d_{1}(f, O)<1$, while $d_{2}(f, O) \geq 2$. Hence $d(f, O) \geq 2$. Here $O$ denotes a representation $(1,0, \ldots, 0)$. Therefore we can distinguish constant, rational and transcendental mmappings by this distance. 
Now,we consider a space of meromorphic mappings

$$
\mathcal{F}:=\left\{f: \mathbb{C}^{m} \rightarrow \mathbb{P}^{n}(\mathbb{C}) \mid \mathrm{f} \text { is meromorphic }\right\} .
$$

In $[4,5,6]$ and this note, a small deformation $\tilde{f}:=L_{2} \cdot h$ of $f$ is represented as

$$
\tilde{f}=\left(h_{0}: h_{1}+a_{1} \zeta_{1} h_{0}:, \ldots . ., h_{n}+a_{n} \zeta_{n} h_{0}\right),
$$

where $h=\left(h_{0}: \cdots: h_{n}\right):=L_{1} \cdot f$. Also, we can choose $\left(a_{1}, \ldots, a_{n}\right)$ such that $\|\mathbf{a}\|:=\left|a_{1}\right|+\cdots+\left|a_{n}\right|$ is as small as possible. So,we can choose $\hat{f}:=L_{1}^{-1} \cdot \tilde{f}$ which is a small deformation without Nevanlinna defects of $f$ such that $d(\hat{f}, f)$ is as small as possible. Hence transcendental meromorphic mappings without Nevanlinna defects are dense in the space of transcendental meromorphic mappings $\mathcal{F}_{0}$.

\section{REFERENCES}

1. A. Edrei and W. H. J Fuchs, Entire and meromorphic functions with asymptotically prescribed characteristic, Canad. J. Math. 17 (1965), 383-395.

2. H. Fujimoto, Non-integrated defect relation for meromorphic maps of complete Kähler manifolds into $\mathbb{P}^{N_{1}}(\mathbb{C}) \times \cdots \times \mathbb{P}^{N_{k}}(\mathbb{C})$, Japan. J. Math. 11 (1985), 233-264.

3. W. K. Hayman, Meromorphic Functions, Oxford, Clarendon, 1964.

4. S. Mori, Elimination of defects of meromorphic mappings of $\mathbb{C}^{m}$ into $\mathbb{P}^{n}(\mathbb{C})$, Ann. Acad. Sci. Fenn. Math. 24 (1999), 89-104.

5. S. Mori, Elimination of defects of meromorphic mappings by small deformation, in: Recent Develpoments in Complex Analisis and Computer Algebra, Proc. of US-Japan Seminar of the Delaware Conference, Kluwer Acad. Publ., 1999, pp. 247-258.

6. S. Mori, Defects of holomorphic curves into $\mathbb{P}^{n}(\mathbb{C})$ for rational moving targets and a space of meromorphic mappings, Complex Variables Theory Appl. vol. 43 (2000), 363-379.

7. R. E. Molzon, B. Shiffman and N. Sibony, Average growth of estimate for hyperplane section of entire analytic sets, Math. Ann. 257 (1987), 43-59.

8. A. Sadullaev, Deficient divisors in Valiron sense, Math. USSR. Sb. 36 (1980), 535547.

9. A. Vitter, The lemma of the logarithmic derivative in several complex variables, Duke Math. J. 44 (1977), 89-104.

Department of Mathematical Sciences, Faculty of Science, Yamagata University

Yamagata, 990-8560 Japan

E-mail: mori@sci.kj.yamagata-u.ac.jp 